$\boldsymbol{B}$ oehmeria Jacq. (Urticaceae) incluye unas 40-50 especies distribuidas principalmente en los trópicos de América y Asia, pero que se extienden a las zonas templadas de Asia, América del Norte, África y Australia (Wilmot-Dear y Friis, 1996). La última revisión del género fue realizada por Wilmot-Dear y Friis (1996), quienes destacaron que Boehmeria y Pouzolzia Gaud. son muy similares, lo que ha conllevado a continuos problemas taxonómicos. En tal sentido, estos géneros son redefinidos como sigue. Boehmeria tiene aquenios romos, amarillos, marrones (cafés) o negros, con la superficie ligeramente escabra, que se dispersan envueltos por el perianto. En el caso de Pouzolzia, el perianto se va desintegrando paulatinamente en los frutos mientras todavía está unido a la infrutescencia y la superficie del aquenio es lisa, usualmente brillante, blanca, café o negra.

Los límites geográficos de la Provincia Biótica de la Península de Yucatán (PBPY) en este trabajo coinciden con los propuestos por EstradaLoera (1991). Así definida, políticamente la PBPY incluye, de norte a sur, los estados mexicanos de Yucatán, Campeche y Quintana Roo, los departamentos de Belize, Corozal y Orange Walk en Belice, y el Departamento del Petén de Guatemala. La inclusión de algunas áreas circunvecinas como la selva Lacandona o la región de Marqués de Comillas en el estado de Chiapas (Durán et al., 1998) es problemática. Estas áreas de Chiapas, aunque presentan el mismo origen geológico que la PBPY, son caracterizadas por un relieve con mayo res elevaciones $\mathrm{y}$ p recipitaciones más abundantes, lo que se refleja en una composición florística distinta.

\title{
NOtAS SOBRE LA FLORA DE LA PENÍNSULA DE YUCATÁN VII: BOEHMERIA CYLINDRICA (URTICACEAE), UN NUEVO REGISTRO PARA
} LA REGIÓN

\author{
José Luis Tapia', Germán Carnevali-Fernández-Concha, \\ Rodrigo Duno-de Stefano, IvÓn M. Ramírez-Morillo, \\ Silvia Hernández-Aguilar y LiLIa LoRena Can-ItZA
}

Herbario CICY, Centro de Investigación Científica de Yucatán, A.C., Calle 43 No. 130, Col. Chuburná de Hidalgo, 97200 Mérida, Yucatán, México. 'Autor para la correspondencia. Correo- e: jltapia@cicy.mx

En un viaje por el sur del estado de Quintana Roo recolectamos un sufrútice urticáceo que a simple vista resultaba una novedad taxonómica para la región. Se estudió el material colectado con la ayuda de una lupa estereoscópica, de la colección general del herbario CICY y la bibliografía existente (e.g. Standley y Steyermark, 1952; Wilmot-Dear y Friis, 1996; Pool, 2001; Steinmann, 2005). Con estas herramientas logramos la identificación inequívoca de los especímenes recolectados como Boehmeria cylindrica (L.) Sw. El género Boehmeria ha sido reportado en varias floras y listados florísticos de América Central (e.g. Standley y Steyermark, 1952; Breedlove, 1986; Balick et al., 2000; Pool, 2001; Correa et al., 2004; Pérez et al., 2005). Sin embargo, Boehmeria nunca había sido reportado en la porción mexicana de la PBPY (Hemsley, 1887; Standley, 1930; Sousa y Cabrera, 1983; Sosa et al.,
1985; Téllez-Valdés y Cabrera-Cano, 1987; Durán et al., 2000; ArellanoRodríguez et al., 2003; GutiérrezBáez, 2003).

Boehmeria cylindrica es una novedad específica para toda la PBPY, aun en su circunscripción más amplia (incluyendo las regiones de La Lacandona y Marqués de Comillas). Nuestras colectas fueron realizadas en la orilla del Río Hondo, por lo cual su presencia en Belice es muy probable. A continuación procedemos a proporcionar una descripción de las plantas de la población de esta especie de la PBPY.

Boehmeria cylindrica (L.) Sw., Prodr. 34. 1788. - Urtica cylindrica L., $S p$. Pl. 2:984. 1753. Figura 1a, b, c y d.

Hierbas o sufrútices, de hasta $1.5 \mathrm{~m}$ de alto; plantas monoicas; tallos cilíndricos o algo angulados, verde pálidos o algo teñidos de rojizo en vida; hojas cartáceas hasta ligera-

Tapia JL, Carnevalli-Fernández-Concha G, Duno-de Stefano R, Ramírez-Morillo IM, Hernández-Aguilar Silvia, Can-Itza LL. 2007. Notas sobre la flora de la Península de Yucatán VII: Boehmeria cylindrica (Urticaceae), un nuevo registro para la región. Boletín de la Sociedad Botánica de México 81: 139-141. 


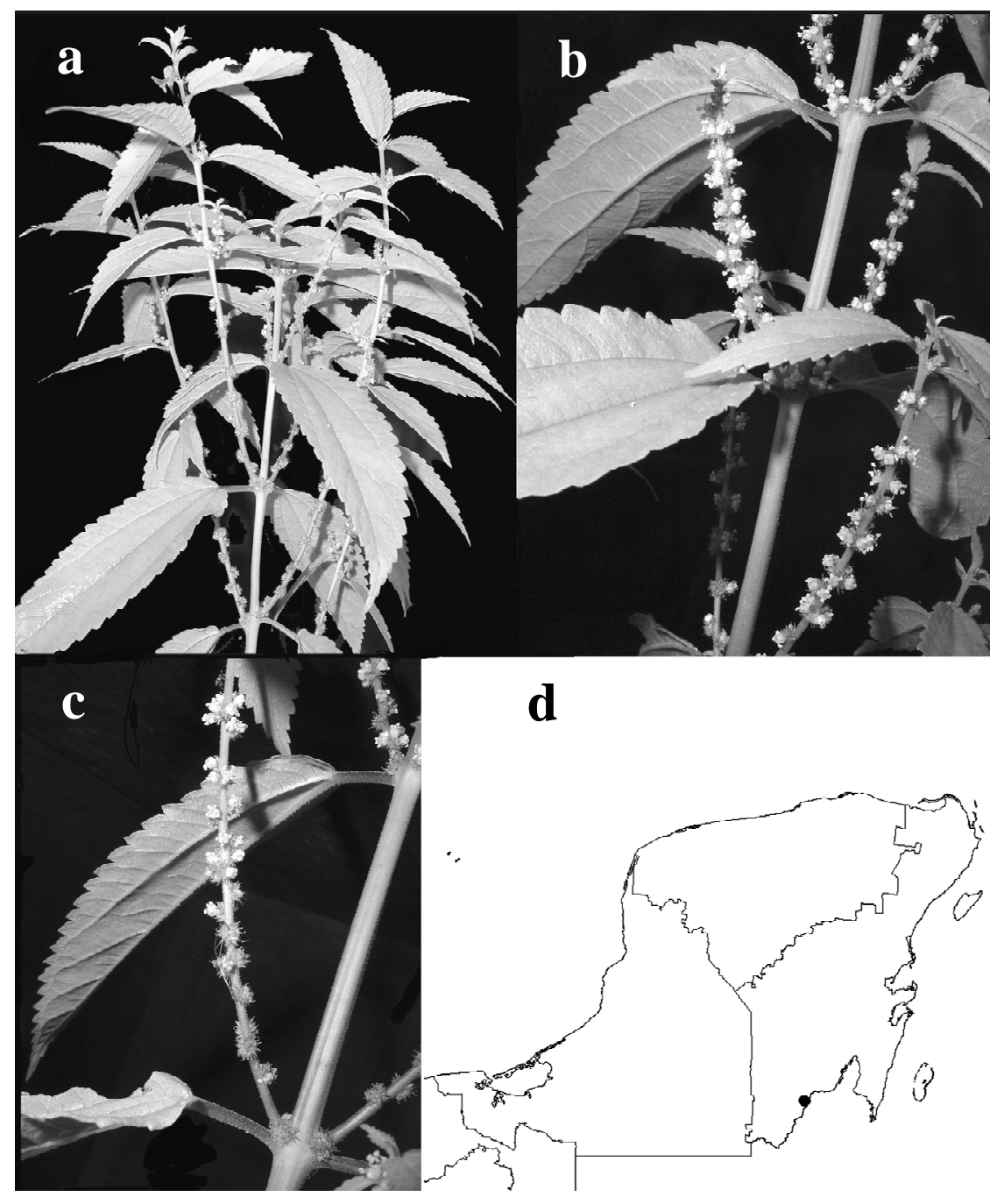

Figura 1a-d. Detalles morfológicos y distribución de Boehmeria cylindrica (L.) Sw. ab. Vista general de hojas e inflorescencia. c. vista general de las inflorescencias axilares y erectas. d. Distribución $(\bullet)$.

mente membranáceas, opuestas o subopuestas, a veces alternas (en las porciones apicales de los tallos), de tamaños similares en nudos adyacentes, estipulas laterales, subuladas, 3-5 $\mathrm{mm}$ de largo, $1 \mathrm{~mm}$ de ancho, rojizas, ligeramente pubescentes en la porción media, caedizas; pecíolos 1-9 cm de largo, estrigulosos, teñidos de rojizo en vida; láminas angostamente ovadas, elípticas a lanceoladas, 4-8 $\mathrm{cm}$ de largo, $1.5-3.5 \mathrm{~cm}$ de ancho, ápice agudo a acuminado, base obtusa a redondeada, a veces ligeramente asimétrica, margen dentado desde la base hasta el ápice, dientes 4-6 mm largo, haz verde oscuro y estrigoso, con cistolitos punctiformes, envés verde claro, suavemente pubescente, pelos adpresos, raramente glabrescentes, conspicuamente trinervadas desde la base, los nervios primarios muy prominentes, de color verde muy pálido hasta casi blancos por el envés, verde oscuro en el haz; inflorescencias en glomérulos en las axilas de las hojas, sésiles, o más frecuentemente en espigas de gloméru- los, unisexuales o bisexuales, 3-10 $\mathrm{cm}$ de largo, erectas, los glomérulos arreglados en forma espiralada, raquis pubescente, cilíndrico a ligeramente angulado, teñido de rojo en vida, generalmente con algunas hojas reducidas en el extremo apical de las espigas, $2.5-4.5 \mathrm{~cm}$ largo, 0.5-1.5 ancho; brácteas florales inconspicuas, 0.3-0.4 mm de largo, rojas, ovadas a orbiculares, apiculadas, pubescentes; flores masculinas 1-3 por glomérulo, principalmente en la porción distal de la espiga, generalmente con perianto 4-partido, algo carnoso, blanco-verdoso a color crema, 0.8$1.2 \mathrm{~mm}$ largo, $1 \mathrm{~mm}$ de diámetro, globoso en botón, esparcidamente adpreso-pubescente, filamentos 0.5 $0.7 \mathrm{~mm}$ de largo, anteras basifijas, blancas, $0.3 \mathrm{~mm}$ de largo, dehiscencia longitudinal; flores femeninas tetrámeras, con perianto fusionado y formando un tubo que envuelve completamente al ovario, $1.3 \mathrm{~mm}$ de diámetro, ovoide, esparcidamente adpreso pubescente, dentado en el ápice, base redondeada a truncada, claramente aplanado dorsoventralmente y alado, verde pálido, teñido de rojo, estigma ca. $0.5 \mathrm{~mm}$ de largo, linearovado a circular; fruto aquenio, cubierto por el perianto persistente, verde pálido o algo teñido de rojo, $c a$. $0.7 \mathrm{~mm}$ de largo, 0.5 de ancho, puberulento, aplanado dorsoventralmente, con alas laterales definidas.

MATERIAL REVISADO: MÉXICO: Quintana Roo: Municipio Othón P. Blanco, Allende, orillas del río Hondo, $14 \mathrm{~km}$ al sur de la desviación a La Unión, por la carretera Chetumal-Escárcega, $22 \mathrm{~km}$ al $\mathrm{O}$ de Chetumal, $18^{\circ} 22^{\prime} 48^{\prime \prime} \mathrm{N}, 88^{\circ} 32^{\prime}$ '5 " O, 5 m s.n.m., 31 de julio 2006, (fl., fr.), J. L. Tapia et al. 1895 (CICY); misma localidad, 17 de enero de 2003 (fl., inmaduras), G. Carnevali 6593 (CICY).

DISTRIBUCIÓN Y ECOLOGÍA: Boehmeria cylindrica se conoce en México en los estados de Coahuila, 
Hidalgo, México, Morelos, Nuevo León, Querétaro, Puebla, Tabasco, Tamaulipas, San Luis Potosí, Veracruz y ahora en Quintana Roo. Así mismo, la especie está ampliamente distribuida en el Nuevo Mundo desde Estados Unidos de América hasta A rgentina y las Antillas. Wilmot-Dear y Friis (1996) mencionan que $B$. cylindrica crece en bosques húmedos, bosques secundarios, frecuentemente a lo largo de cursos de agua, en áreas húmedas del bosques seco, en áreas intervenidas, herbazales, pantanos y como maleza en plantaciones, entre 30-250 m s.n.m. En nuestro caso, los dos especímenes fueron recolectados en las márgenes del río Hondo, en áreas intervenidas muy húmedas asociadas a un bosque húmedo ribereño, a $5 \mathrm{~m}$ s.n.m. En la misma localidad y microambientes se colectaron plantas tales como Montrichardia arborescens (L.) Schott (Araceae), Pavonia schiedeana Steud. (Malvaceae) y varias cyperáceas.

DISCUSIÓN: Boehmeria cylindrica es morfológicamente similar a B. cau data $\mathrm{Sw}$. en el arreglo de su inflorescencia y en la forma del fruto, pero $B$. cylindrica es fácilmente distinguible por sus inflorescencias erectas ( $v s$. péndulas), frutos estrechamente delgados y alados, hojas más membranáceas y los márgenes con dientes desde la base hasta el ápice (Wilmot-Dear y Friis 1996). Además, B. caudata ocupa pisos altitudinales superiores y apenas se solapa con el intervalo altitudinal de B. cylindrica (sólo en México, América Central y parte de Brasil). El tratamiento de la familia Urticaceae para El Bajío y de regiones adyacentes (Steinmann, 2005) incluye un excelente dibujo de $B$. cylindrica.

Recibido: 4 de diciembre de 2006 Aceptado: 31 de julio de 2007

\section{Literatura Citada}

Arellano-Rodríguez J.A., Flores-Guido J.S., Tun-Garrido J. y CruzBojórquez M.M. 2003. Nomenclatura, Forma de Vida, Uso, Manejo y Distribución de las Especies Vegetales de la Península de Yucatán. Etnoflora Yucatanense, Fascículo 20. Unive rsidad Autónoma de Yucatán, Mérida.

Balick M.J., Nee M.H. y Atha D.E. 2000. Checklist of the vascular plants of Belize, with common names and uses. Memories of the New York Botanical Garden 85:1-246.

Breedlove D.E. 1986. Listados florísti cos de México IV. Flora de Chiapas. Instituto de Biología, Universidad Nacional Autónoma de México, México, D.F.

Correa-A. M.D., Galdames C. y Sánchez de Stapf M. 2004. Catálogo de las Plantas Vasculares de Panamá. Universidad de Panamá, Instituto de Investigaciones Tropicales, Autoridad Nacional del Ambiente y Corredor Biológico Mesoamericano del Atlántico Panameño, Panamá.

Estrada-Loera E. 1991. Phytogeographic relationships of the Yucatan Peninsula. Journal of Biogeography 18:687-697.

Durán R., Trejo-Torres J.C. e IbarraManríquez G. 1998. Endemic phytotaxa of the Peninsula of Yucatán. Harvard Papers in Botany 3:263-316.

Durán R., Campos G., Trejo J.C., Simá P., May-Pat F. y Juan-Qui M. 2000. Listado Florístico de la Península de Yucatán. Centro de Investigación Científica de Yucatán, Mérida.

Gutiérrez-Báez, C. 2003. Listado Florístico Actualizado del Estado de Campeche, México. Universidad Autónoma de Campeche, Campeche.

Hemsley W.B. 1887. Cozumel Island. Enumeration of a small collection of plants made in 1885 by F. Gaumer. En: Supplement, Biologia Centrali Americana, Botany 4: 96-114.
Pérez A.L., Sousa-S. M., Hanan A.M., Chiang F. y Tenorio P. 2005. Vegetación Terrestre. En: Bueno J., Álvarez F. y Santiago S. (Eds.). Biodiversidad del Estado de Tabasco, pp. 65-110. Instituto de Biología, Universidad Nacional Autónoma de México y Comisión Nacional para el Conocimiento y Uso de la Biodiversidad, México, D.F.

Pool A. 2001. Urticaceae. En: Stevens W.D., Ulloa C., Pool A. y Montiel M.O. (eds.). Flora de Nicaragua. Monographs in Systematic Botany from the Missouri Botanical Garden 85:2479-2495.

Sosa V., Flores J.S., Rico-Gray V., Lira R. y Ortiz J.J. 1985. Lista Florística y Sinonimia Maya. En: Etnoflora Yucatanense, Fascículo 1. Instituto Nacional de Investigaciones sobre Recursos Bióticos, Xalapa.

Sousa M. y Cabrera E.F. 1983. Listados Florísticos de México. II. Flora de Quintana Roo. Instituto de Biología, Universidad Nacional Autónoma de México, México, D.F.

Standley P.C. 1930. Flora of Yucatan. Field Museum of Natural History Botanical Series 3:157-491.

Standley P.C. y Steyermark J.A. 1952. Urticaceae. En: Flora of Guatemala (Standley, P. C. y J. A. Steyermark, eds.). Fieldiana Botany 24:396-430.

Steinmann, V.W. 2005. Urticaceae. Fascículo No. 134. En: Rzedowski J. y Calderón G. Eds. Flora del Bajío y de Regiones Adyacentes, pp. 1-74, Instituto de Ecología, A.C., Centro Regional del Bajío, Pátzcuaro.

Téllez-Valdés O. y Cabrera-Cano E.F. 1987. Listados Florísticos de Mexico VI. Flórula de la isla de Cozumel, Q. $R$. Instituto de Biología, Instituto de Biología, Universidad Nacional Autónoma de México, México, D.F.

Wilmot-Dear C.M. y Friis I. 1996. The new world species of Boehmeria and Pouzolzia (Urticaceae, tribu Boehmerieae). A taxonomic revision. Opera Botanica 129:1-103. 